\title{
INTRACERVICAL FIBROID REMOVAL: A MYOMECTOMY REASONED ON BIOLOGICAL BASES.
}

\author{
Andrea Tinelli ${ }^{1}$ \\ ${ }^{1}$ Presidio Ospedaliero Vito Fazzi
}

August 11, 2021

Commentary on: LAPAROSCOPIC MYOMECTOMY USING LOOP LIGATION FOR GIANT INTRACERVICAL MYOMAS: A NOVEL SURGICAL TECHNIQUE.

Authors: Shengke Wang, Dongdong Wang, Qihong Huang, Fujie Zhao.

Journal: BJOG: An International Journal of Obstetrics \& Gynaecology

INTRACERVICAL FIBROID REMOVAL: A MYOMECTOMY REASONED ON BIOLOGICAL BASES .

Dr. Andrea Tinelli, MD, Prof, PhD

Veris delli Ponti Hospital, Obstetrics and Gynecology Department, Scorrano, Lecce, Italy; Laboratory of Human Physiology,Phystech BioMed School, Faculty of Biological \& Medical Physics,Moscow Institute of Physics and Technology (State University), Dolgoprudny, Moscow Region, Russia.

Tel: +39-3392074078; E-mail:andreatinelli@gmail.com; ORCID: 0000-0001-8426-8490

Anatomically, uterine corpus, isthmus and cervix compose one organ, but functionally they attend different function during pregnancy and labor. The uterine cervix is mainly composed connective tissue and extracellular matrix, that allow the pregnancy to come to an end, up to the onset of labor, when cervical ripening and dilatation occur to deliver the fetus. The cervical innervations and the different neurotransmitters and neuropeptides expression involved in cervical ripening suggest that the cervix plays a key role in pregnancy maintenance, labor initiation, pain and delivery; this can also be supported by previous studies that showed that cervical ripening is also a neuroimmune-mediate inflammatory reaction involveing the hypogastric nerve [Di Tommaso S "et al", 2017;18(2):140-148].

Neuropeptides are signaling peptides that are produced by neural, endocrine and/or immune cells: all of these hormones are involved in a variety of biological processes, not only enhancing uterine contractility and modulating pain trigger, but also possessing anti-inflammatory, antioxidative stress and tumorigenic properties. Particularly, they contribute with changes in muscle contractility, uterine peristalsis and muscular healing and may be involved also in the uterine fibroids' pathophysiology [Tinelli A "et al"; 2020;21(5):440442].

Uterine fibroids are generally distributed over the body and fundus of the uterus, they are surrounded by a fibroneurovascular network rich of neurotransmitters, the myoma pseudocapsule, a neurovascular bundle separating fibroid from the myometrium, and allowing less bleeding during myomectomy and better subsequent myometrial healing after removal [Tinelli A "et al"; Curr Protein Pept Sci. 2017;18(2):129-139]. For this reason, the correct myomectomy which enucleates fibroid inside its pseudocapsule has been called "intracapsular" and has remarkable early and late biological - muscular advantages, during and after surgery [Tinelli A "et al".; JSLS 2012;16(1):119-29]. 
Cervical fibroids are infrequent, but often create significant problems during myomectomy, as, during the removal there can be intraoperative and late complications, such as massive bleeding and scarring fibrosis with all the repercussions on pregnancy and childbirth [Malvasi A "et al"; 2013;29(11):982-8.].

Wang "et al" [Wang "et al"; BJOG 2021] proposed their intracervical myomectomy for large intracervical myomectomy on 12 patients, basing on biology of the myoma pseudocapsule. They performed a laparoscopic myomectomy putting a loop ligation along the junction of the pseudocapsule and cervix, pulling the loop it at this position; then, they performed a traction and enucleation within the fibroid wound cavity directly closes the fibroid cavity, with the surrounding vascular network bounded in the knot after ligation of the pseudocapsule without dead space, and preventing injury to nearby tissues.

During fibroid enucleation, loop ligation of the pseudocapsule glides along the tumor body and operates within the fibroid wound cavity, with no cervical tissue involved during enucleation. The neurovascular bundles of the fibroid pseudocapsule are protected and spared during myomectomy and the side of the pseudocapsule attached to the fibroid was bound tightly in the loop to achieve hemostasis. This technique results less invasive and would promote fertility in case of cervical myomectomy, not requiring additional pelvic dissection and allowing to operate directly in the cervix sparing adjacent tissues and pseudocapsule. 